\section{References}

1. Lima CKT, Carvalho PMM, Lima IAAS, et al. The emotional impact of coronavirus 2019-nCOV (new coronavirus disease). Phychiatry Res 2020;287: 112915.

2. Li W, Yang Y, Liu $\mathrm{ZH}$, et al. Progression of mental health service during COVID-19 outbreak in China. Int J Biol Sci 2020;16:1732-1738.
3. Lu W, Wang H, Lin Y, Li L. Psychological status of medical workforce during the COVID-19 pandemic: a cross-sectional study. Psychiatry Res 2020; 288:112936.

4. Spitzer RL, Kroenke K, Williams JB, Lowe B. A brief measure for accessing generalized anxiety disorder: the GAD-7. Arch Intern Med 2006;166: 1092-1097.

5. Bruns DP, Kraguljac NV, Bruns TR. COVID-19: facts, cultural consideration and risk of stigmatization. J Transcult Nurs 2020;31:326-332.

\title{
May we learn a useful lesson from prevention rules against severe acute respiratory coronavirus virus 2 (SARS-CoV-2)?
}

\author{
Silvia Corbellini MD, Maria Antonia De Francesco PhD and Arnaldo Caruso PhD \\ Department of Molecular and Translational Medicine, Institute of Microbiology, University of Brescia-ASST Spedali Civili di Brescia, Brescia, Italy
}

To the Editor-The emergence and diffusion of oxacillin-resistant Staphylococcus aureus (MRSA) constitutes an important problem for public health. Data from European countries reported a trend with an increasing MRSA prevalence from the north to the south of the continent: $<5 \%$ of MRSA has been isolated from invasive infections in north of Europe compared with $25 \%-50 \%$ in the south of Europe. ${ }^{1}$

This gram-positive bacterium is generally found as part of commensal flora in the nasal mucosa in $20 \%-40 \%$ of the population and just these people, who are asymptomatic carriers, have an increased risk to acquire a subsequent infection in addition to representing an important source of person-to-person transmission. In particular, hospital and healthcare settings represent a favorable environment that predispose to infection because of a high antibiotic selection pressure, the use of invasive procedures, and the presence of critically ill patients. For these reasons, MRSA is now endemic in many hospitals worldwide, and infection control measures are needed to prevent its transmission, especially considering the risk of development of glycopeptide-resistant $S$. aureus strains.

Hospital control of endemic MRSA has been based on standard precautions such as isolation/cohorting, hand hygiene, patient decolonization, and appropriate use of antibiotic (antibiotic stewardship). Intensive care units of Spedali Civili's Hospital of Brescia has implemented active surveillance cultures to identify patients who acquire MRSA during hospitalization. This surveillance involves nasal swabs for the screening of patients at the time of hospital admission to identify asymptomatic carriers, followed by periodic screening every 3 days.

One of these intensive care units became a coronavirus disease 2019 (COVID-19) ward during the pandemic, and we analyzed whether the higher compliance to the use of personal protective equipment (PPE, eg, gloves, coveralls, face mask and boots) by all the hospital staff had an impact on the prevalence of MRSA acquisition during patients hospitalization.

Author for correspondence: Arnaldo Caruso, E-mail: arnaldo.caruso@unibs.it

Cite this article: Corbellini S, De Francesco MA, and Caruso A. (2021). May we learn a useful lesson from prevention rules against severe acute respiratory coronavirus virus 2 (SARS-CoV-2)? Infection Control \& Hospital Epidemiology, 42: 1290-1291, https:// doi.org/10.1017/ice.2020.1228
Table 1. Trend of MRSA Detection During the Study Period

\begin{tabular}{lcccc} 
& 2019 & & 2020 & \\
\cline { 2 - 2 } Year & No. of Positive/Total (\%) & & No. of Positive/Total (\%) & $P$ Value \\
\hline January & $9 / 103(8)$ & & $4 / 134(3)$ & .08 \\
\hline February & $10 / 102(9)$ & $2 / 126(2)$ & .01 \\
\hline March & $18 / 135(12)$ & $5 / 274(2)$ & .0001 \\
\hline April & $13 / 108(11)$ & $7 / 269(3)$ & .01 \\
\hline May & $25 / 97(20)$ & $4 / 193(2)$ & .0001 \\
\hline June & $21 / 83(20)$ & $2 / 178(1)$ & .0001 \\
\hline July & $17 / 97(15)$ & $0 / 183(0)$ & .0001 \\
\hline August & $21 / 107(16)$ & $8 / 153(5)$ & .01 \\
\hline Total & $134 / 832(14)$ & $32 / 1510(2)$ & .0001 \\
\hline
\end{tabular}

It is well known that healthcare workers can transmit infections such as tuberculosis, varicella and influenza by the airborne route, ${ }^{2}$ but it less well known that airborne and other ways of transmission may occur with some bacterial pathogens. In particular, the use of face masks prevents pathogen transmission from the wearer to other people and reduces hand-to-face contact and facial contact with droplets. ${ }^{3}$

In our analysis, we compared the MRSA detection after 48 hours following hospital admission during January-August 2020 versus January-August 2019. As shown in Table 1, we observed a statistically significant reduction in the prevalence of nosocomially acquired MRSA ( $2 \%$ vs $14 \% ; P<.0001)$. This decrease was always statistically significant for all the months analyzed except January, when the implementation of PPE in the absence of COVID-19 was not present (Table 1). This finding is not surprising, since a previous study showed that a healthcare worker, who did not wear a mask and who was a nasal carrier of MRSA, induced a 40-fold increase in MRSA dispersion. ${ }^{4}$

Although our preliminary data need to be confirmed by larger studies, our observation suggests implementation of PPE as a strong preventive strategy to control hospital-acquired MRSA infection. 


\section{Acknowledgments.}

Financial support. No financial support was provided relevant to this article.

Conflicts of interest. All authors report no conflict of interest relevant to this article.

\section{References}

1. European Antimicrobial Resistance Surveillance Network (EARS-Net). European Centre for Disease Prevention and Control website. http://
ecdc.europa.eu/en/activities/surveillance/EARS-Net/Pages/index.aspx. Published 2018.

2. Weber DJ, Rutala WA, Schaffner W. Lesson learned: protection of healthcare workers from infectious disease risks. Crit Care Med 2010;38:S306S314.

3. Radonovich LJ Jr, Simberkoff MS, Bessesen MT, et al. N95 respirators vs medical masks for preventing influenza among health care personnel: a randomized clinical trial. JAMA 2019;322:824-833.

4. Sherertz RJ, Reagan DR, Hampton KD, et al. A cloud adult: the Staphylococcus aureus virus interaction revised. Ann Intern Med 1996;124:539-547.

\title{
Reduction in ambulatory visits for acute, uncomplicated bronchitis: an unintended but welcome result of the coronavirus disease 2019 (COVID-19) pandemic
}

\author{
Thomas J. Dilworth PharmD (1) and Charles F. Brummitt MD \\ AdvocateAuroraHealth, Milwaukee, Wisconsin
}

To the Editor-The coronavirus disease 2019 (COVID-19) pandemic has adversely impacted nearly all aspects of life since emerging in late 2019. Notably, surges in COVID-19 cases have led to antibiotic overprescribing in the inpatient setting, hampering ongoing antimicrobial stewardship efforts. ${ }^{1,2}$ However, patients' reluctance to seek healthcare during the pandemic, particularly for minor ailments, may lead to unexpected outpatient antimicrobial stewardship gains. Jeffery et $\mathrm{al}^{3}$ reported an inverse relationship between COVID-19 cases and daily counts of emergency department visits in 5 US states between January and April of 2020. Diagnoses were not reported in this study, and the authors concluded that clinicians should reinforce to patients the importance of seeking emergency department care for serious conditions. We have previously reported the results of a systemwide initiative to reduce antibiotic prescribing for ambulatory adults with acute, uncomplicated bronchitis. ${ }^{4}$ Internally, we continue to track and report these data. Recently, we observed a profound reduction in both the overall number of patients seen and discharged with a primary diagnosis of bronchitis and the number of antibiotic prescriptions written for these encounters (Fig. 1). In addition to patients' not seeking care due to the pandemic, there are a number of other, possible explanations for our observed decline in visits. Stay-at-home orders and social distancing appear to have reduced the burden of common respiratory viruses in the community, leading to fewer cases of acute, uncomplicated bronchitis, ${ }^{5,6}$ for which the primary etiology is viral. ${ }^{7}$ Patients may increasingly seek care outside of our health system during the pandemic. The pandemic has also shifted patient care to telemedicine. To assess this phenomenon, we captured whether or not a visit was a telemedicine encounter and then analyzed the recent ambulatory clinic data. Of the 394 ambulatory clinic bronchitis visits during July and August, $112(28.4 \%)$ were telemedicine encounters, a higher proportion of

Author for correspondence: Thomas J. Dilworth, E-mail: thomas.dilworth@aah.org Cite this article: Dilworth TJ and Brummitt CF. (2021). Reduction in ambulatory visits for acute, uncomplicated bronchitis: an unintended but welcome result of the coronavirus disease 2019 (COVID-19) pandemic. Infection Control \& Hospital Epidemiology, 42: 1291-1292, https://doi.org/10.1017/ice.2020.1233 total visits than observed in previous months. Thus, a shift toward more telemedicine visits was revealed in our data set.

The COVID-19 pandemic, while challenging for so many other aspects of antimicrobial stewardship, has led to an overall net
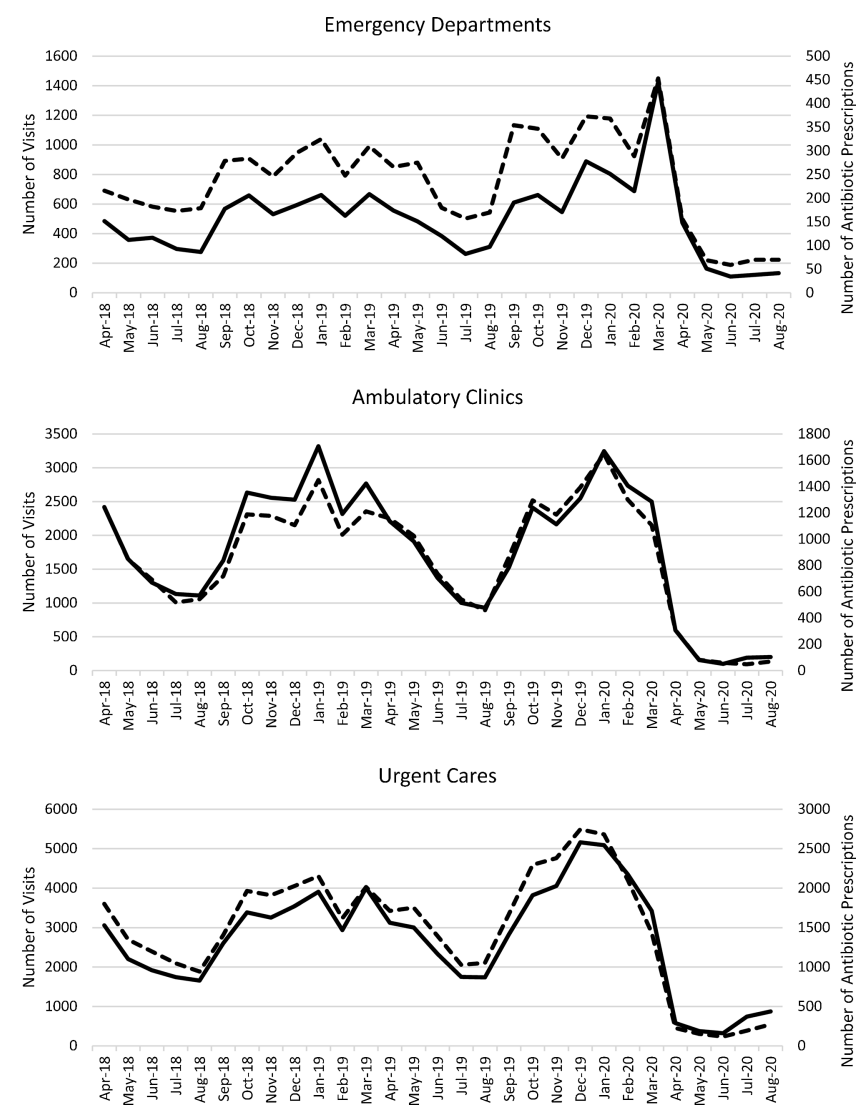

Fig. 1. Number of ambulatory patients with a primary diagnosis of bronchitis, April 2017-August 2020. Complete lines, number of visits; dashed lines, number of antibiotic prescriptions. 